\title{
Breast Cancer and Hormonal Stimulation: Is Glycolysis the First Sign of Response?
}

$\mathbf{T}$ he estrogen receptor (ER) is a critical therapeutic target for breast cancer. Nearly $70 \%$ of newly diagnosed breast cancers express ER, and frequent dependence of breast cancer on estrogen stimulation for growth makes endocrine therapy an attractive choice for breast cancer treatment. Multiple therapies, including tamoxifen (a partial agonist and antagonist of ER), aromatase inhibitors, and selective ER downmodulators and estradiol itself, provide effective treatment for this common cancer, typically with considerably fewer side effects than cytotoxic chemotherapy.

The molecular basis of ER-stimulated growth in breast cancer is

\section{See page 1740}

increasingly well characterized. Estradiol from the blood, or possibly produced in the tumor itself, binds to the $\mathrm{ER}$, a nuclear receptor, resulting in receptor dimerization. In concert with an ensemble of cofactors, the dimerized ER interacts with specific genomic segments, known as estrogen-response elements, resulting in the transcription of genes that perform the estrogen response. In breast cancers, a key response to estrogen is an increase in cellular proliferation. Some more recent studies have suggested that there may also be a response mediated by nonnuclear ER located in the cell membrane or cytosol. Nonnuclear ER

Received Jun. 19, 2010; revision accepted Jul. 2, 2010.

For correspondence or reprints contact: Hannah M. Linden, Seattle Cancer Care Alliance and University of Washington, 825 Eastlake Ave. East, Seattle, WA 98109.

E-mail: hmlinden@u.washington.edu

COPYRIGHT (C) 2010 by the Society of Nuclear

Medicine, Inc.

DOI: 10.2967/jnumed.110.078329 may play an important role in the interaction of the estrogen response with other growth factor pathways such as the epidermal growth factor pathway $(1,2)$.

Because not all breast cancer patients respond to ER-directed therapy, predicting therapeutic response to endocrine treatment is an important clinical need. Only patients whose tumors express ER, or possibly the closely related progesterone receptor, respond to endocrine therapy; response rates for patients with ER-negative tumors are generally less than $5 \%$. The determination of ER expression by in vitro assay, and possibly also by imaging (3), is the first step in selecting breast cancer patients for endocrine therapy and reserving treatment only for those patients with ERexpressing $(\mathrm{ER}+)$ breast tumors.

However, not all ER+ tumors respond to endocrine treatment. Only $50 \%-75 \%$ of untreated patients with $\mathrm{ER}+$ tumors respond, and considerably fewer who have already failed an endocrine treatment will respond to an alternative ER-directed drug. Therefore, in addition to determining which patients have ER expression, there is an equally important need to predict which ER+ tumors will respond to endocrine therapy. One welltested approach is to have patients undertake a short treatment with an endocrine agent and then to assay changes in tumor growth. This approach has been best studied and validated using serial tumor biopsy and the in vitro assay of cellular proliferation, typically the $\mathrm{Ki}-67$ index, as a measure of response $(4,5)$. Data from the Immediate Preoperative Arimidex Compared with Tamoxifen trial demonstrated that a low Ki-67 2 weeks after initiation of endocrine treatment predicts benefit from endocrine ther- apy and a low risk of relapse. Longterm follow-up of patients undergoing neoadjuvant endocrine therapy confirmed these findings and demonstrated an extremely favorable outcome for patients downstaged to stage I after 4 mo of endocrine treatment (6). These results have led to increasing acceptance of the 2-week $\mathrm{Ki}-67$ index as a predictive marker for endocrine therapy; however, this approach is invasive, is subject to sampling error, and can be challenging to implement in the clinic.

The innovative work of Dehdashti et al. at Washington University demonstrated that, like biopsy, serial ${ }^{18}$ F-FDG PET was highly predictive of breast cancer endocrine response but, unlike biopsy, was noninvasive and clinically feasible (7-9). The group based their approach on the observation that patients who experience tumor-related symptoms or flare in response to transient estrogen stimulation are also likely to respond to endocrine therapy. The Washington University investigators showed that an early increase in ${ }^{18} \mathrm{~F}$-FDG uptake in response to an estrogen agonist, seen as soon as $1-2 \mathrm{~d}$ after administration, predicted a response to endocrine therapy and subsequent patient outcomes. On the basis of these results and the serial biopsy studies in aromatase inhibitors, we surmised that the withdrawal of estrogens after aromatase inhibitor therapy would result in an early decline in ${ }^{18} \mathrm{~F}-\mathrm{FDG}$ uptake, a hypothesis that has been supported by early results (10). Taken together, these observations indicate that glycolysis, as measured by ${ }^{18} \mathrm{~F}$ FDG uptake, is a pharmacodynamic marker of breast cancer response to the addition or withdrawal of estrogenic stimulation, suggesting a potential role in selecting breast cancer patients for endocrine treatment. 
The underlying presumption in these studies, perhaps unstated, is that the increase in glycolysis in response to estrogen stimulation is simply the result of increased cellular proliferation, a known effect of estrogens in responsive breast cancers. The article by Ko et al. (11), in this issue of The Journal of Nuclear Medicine, may shed some light on the mechanism of increased ${ }^{18}$ F-FDG uptake after estradiol administration and suggests that the answer is not as simple as an increase in cell growth. Using breast cancer cell lines, the authors present data that suggest that increased ${ }^{18} \mathrm{~F}$ FDG uptake occurring after estrogen stimulation is mediated by nonnuclear ER. An estrogen construct that has access to the cell membrane, but not the nucleus, had effects similar to those of estradiol on ${ }^{18} \mathrm{~F}$-FDG uptake. In accordance with in vivo studies from Washington University (8), Ko et al. found increased ${ }^{18} \mathrm{~F}$-FDG uptake with early exposure to tamoxifen. They found that increased ${ }^{18} \mathrm{~F}-\mathrm{FDG}$ uptake correlated with mitochondrial hexokinase activity but was independent of change in glucose transporter type 1 expression. By analyzing protein phosphorylation and using selective inhibitors, the authors showed that the increase in glycolysis with estrogen stimulation was associated with increased activity in the PI3 kinase/ Akt pathway, a pathway important in mediating resistance to cell death. These findings suggest that the increase in glycolysis, and the resulting increase in ${ }^{18} \mathrm{~F}-\mathrm{FDG}$ uptake, in estrogen agonist studies is not simply the result of the changes in cellular proliferation seen in the biopsy-based studies.

The results of this intriguing study raise several important questions. Do findings in cell lines in the controlled environment of the petri dish necessarily hold for highly heterogeneous human breast cancers in the complex in vivo environment of the human host?

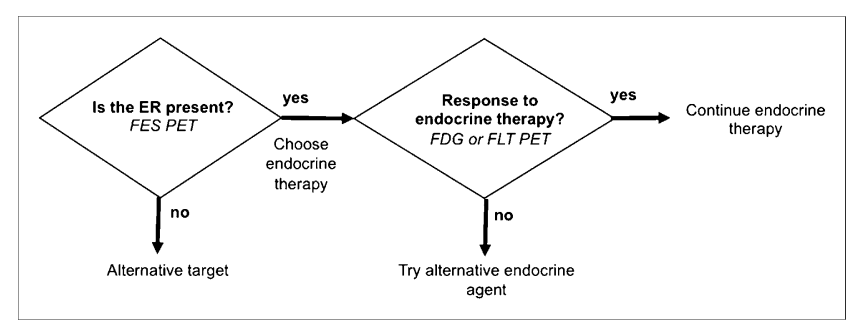

FIGURE 1. Schematic of approach to determining role of molecular imaging in directing individualized endocrine therapy. FES $=16 \alpha-{ }^{18} \mathrm{~F}$-fluoro-17 $\beta$-estradiol.

Are glycolysis and proliferation equivalent predictors of endocrine responsiveness? Would PET probes of proliferation, for example, ${ }^{18} \mathrm{~F}$-fluorothymidine, produce results similar to those found with ${ }^{18}$ F-FDG? Does a change in glycolysis always accompany a change in proliferation? If increased glycolysis is the result of activation of the PI3 kinase pathway, might an ${ }^{18} \mathrm{~F}$ FDG flare be a marker for response to agents that target this pathway? Promising early results using ${ }^{18} \mathrm{~F}-\mathrm{FDG}$ PET as a marker of endocrine responsiveness, combined with these new in vitro results, call for mechanistic studies in humans to determine how ${ }^{18} \mathrm{~F}-\mathrm{FDG}$, or ${ }^{18} \mathrm{~F}$ fluorothymidine, might work as an in vivo predictive assay for breast cancer endocrine therapy. Clinical trials should also be done to determine the role that molecular imaging might play in directing individualized endocrine therapy, guided by the approach illustrated in Figure 1. Early results suggest a potentially important role for molecular imaging in the management of $\mathrm{ER}+$ breast cancer and clearly merit further study.

\section{ACKNOWLEDGMENT}

This work was supported in part by $\mathrm{NIH}$ grant $\mathrm{CA} 42045, \mathrm{NIH} / \mathrm{NCI}$ 1RC1CA146456, and Avon-NCI 3 P30 CA015704-34S3.

\section{Hannah M. Linden \\ David A. Mankoff \\ University of Washington and Seattle \\ Cancer Care Alliance \\ Seattle, Washington}

\section{REFERENCES}

1. Johnston SR. New strategies in estrogen receptorpositive breast cancer. Clin Cancer Res. 2010; 16:1979-1987.

2. Arpino G, Wiechmann L, Osborne CK, Schiff R. Crosstalk between the estrogen receptor and the HER tyrosine kinase receptor family: molecular mechanism and clinical implications for endocrine therapy resistance. Endocr Rev. 2008;29: 217-233.

3. Peterson LM, Mankoff DA, Lawton T, et al. Quantitative imaging of estrogen receptor expression in breast cancer with PET and ${ }^{18} \mathrm{~F}$-fluoroestradiol. J Nucl Med. 2008;49:367-374.

4. Dowsett M, Smith IE, Ebbs SR, et al. Prognostic value of Ki67 expression after short-term presurgical endocrine therapy for primary breast cancer. J Natl Cancer Inst. 2007;99:167-170.

5. Dowsett M, Smith IE, Ebbs SR, et al. Short-term changes in Ki-67 during neoadjuvant treatment of primary breast cancer with anastrozole or tamoxifen alone or combined correlate with recurrence-free survival. Clin Cancer Res. 2005;11(2 Pt 2):951s958s.

6. Ellis MJ, Tao Y, Luo J, et al. Outcome prediction for estrogen receptor-positive breast cancer based on postneoadjuvant endocrine therapy tumor characteristics. J Natl Cancer Inst. 2008;100:13801388.

7. Dehdashti F, Mortimer JE, Trinkaus K, et al. PETbased estradiol challenge as a predictive biomarker of response to endocrine therapy in women with estrogen-receptor-positive breast cancer. Breast Cancer Res Treat. 2009;113:509-517.

8. Mortimer JE, Dehdashti F, Siegel BA, Trinkaus K, Katzenellenbogen JA, Welch MJ. Metabolic flare: indicator of hormone responsiveness in advanced breast cancer. J Clin Oncol. 2001;19:2797-2803.

9. Ellis MJ, Gao F, Dehdashti F, et al. Lower-dose vs high-dose oral estradiol therapy of hormone receptorpositive, aromatase inhibitor-resistant advanced breast cancer: a phase 2 randomized study. JAMA. 2009;302: 774-780.

10. Linden H, Kurland B, Livingston R, et al. Early assessment of response to aromatase inhibitor (AI) therapy [abstract]. J Clin Oncol. 2009;27 (suppl):15s.

11. Ko B-H, Paik J-Y, Jung K-H, Lee K-H. 17ßEstradiol augments ${ }^{18} \mathrm{~F}$-FDG uptake and glycolysis of T47D breast cancer cells via membrane-initiated rapid PI3K-Akt activation. J Nucl Med. 2010;51: 1740-1747. 